\title{
A Difficulty in Williams' Algorithm for Interpolating Rationals
}

\author{
By Charles B. Dunham
}

\begin{abstract}
J. Williams has developed a theory and an algorithm for best Chebyshev approximation of decay-type functions by an oscillation factor times a negative power of a linear form (in particular a polynomial). It is shown that the levelling equations of the algorithm may not have an admissible solution.
\end{abstract}

The problem of Williams is as follows. Let $\alpha>0$ and $C[0, \alpha]$ be the space of continuous functions on $[0, \alpha]$. For $h \in C[0, \alpha]$, define

$$
\|h\|=\max \{|h(x)|: 0 \leqslant x \leqslant \alpha\} .
$$

Let $B \in C[0, \alpha]$ and $g$ be a positive element of $C[0, \alpha]$. Let $\left\{\phi_{1}, \ldots, \phi_{n}\right\}$ be a Chebyshev set on $[0, \alpha]$ and define

$$
L(A, x)=\sum_{k=1}^{n} a_{k} \phi_{k}(x)
$$

Let $p>0$. The problem of Williams is to find a coefficient vector $A^{*}$ to minimize $\left\|B\left(g-[L(A, \cdot)]^{-p}\right)\right\|$ over $\{A: L(A, \cdot)>0\}$.

Williams gives an algorithm, which involves solution of the system of error-levelling equations

$$
\left|B\left(x_{i}\right)\right|\left(g\left(x_{i}\right)-\left[L\left(A, x_{i}\right)\right]^{-p}\right)=(-1)^{i} \lambda, \quad i=1, \ldots, n+1 .
$$

Williams claims that a solution exists if $B\left(x_{i}\right) \neq 0$ for $i=1, \ldots, n+1$. It is shown below that this solution may not be admissible.

THEOREM. Let $L(C, \cdot)$ change sign on $[0, \alpha]$ and be positive on a subinterval $I$ on which $B$ is nonzero. Let $x_{1}, \ldots, x_{n+1}$ be distinct points in $I$. There exists $g>0$ such that $(C, 0)$ is a solution to (1) and no pole-free solution exists.

Proof. Choose $g$ such that $g\left(x_{i}\right)=\left[L\left(C, x_{i}\right)\right]^{-p}$, then $(C, 0)$ is a solution to (1). Suppose $(A, \lambda)$ is another solution with $L(A, \cdot)>0$. Then

$$
\left|B\left(x_{i}\right)\right|\left(\left[L\left(C, x_{i}\right)\right]^{-p}-\left[L\left(A, x_{i}\right)\right]^{-p}\right)=(-1)^{i} \lambda, \quad i=0, \ldots, n+1 .
$$

As we are assuming that $L(A, \cdot), L(C, \cdot)$ are positive on $I$, and $B$ is of constant sign on $I$, this implies that $L(A, \cdot)-L(C, \cdot)$ alternates in sign on $\left\{x_{1}, \ldots, x_{n+1}\right\}$ and, hence, 
has $n$ zeros on $I$, contradicting the Chebyshev set hypothesis on the basis.

It seems that the best that can be proven is that Williams' algorithm converges if the starting points are sufficiently close (as in [1]). The approach of [1] can be used to prove this.

In running Williams' algorithm for approximation of $\exp (-x)$ on $[0,10]$ with $p=1, B=1$, the author did encounter approximations with poles. The poles disappeared on iteration, but this may not occur in general.

Computer Science Department

University of Western Ontario

London, Ontario, Canada

1. R. BARRAR \& H. L. LOEB, "On the Remez algorithm for non-linear families," Numer. Math., v. 15, 1970, pp. 382-391. MR 42 \#2626.

2. J. WILLIAMS, "Numerical Chebyshev approximation by interpolating rationals," Math.

Comp., v. 26, 1972, pp. 199-206. 